\title{
A 36-Year-Old Moroccan Man Presenting with Widespread Papules and Pustules
}

\author{
Anna Morenz $^{a}$ Sherry H. Yu ${ }^{b, c} \quad$ Kristine Cornejo $^{d} \quad$ Bonnie Mackool $^{c}$ \\ a Department of Medicine, University of Washington, Seattle, WA, USA; \\ ${ }^{b}$ Harvard Combined Dermatology Residency Program, Boston, MA, USA; \\ 'Department of Dermatology, Massachusetts General Hospital, Boston, MA, USA; \\ ${ }^{\mathrm{d} D e p a r t m e n t}$ of Pathology, Massachusetts General Hospital, Boston, MA, USA
}

\author{
Keywords \\ Papulonecrotic tuberculosis · Infectious disease
}

\section{Abstract}

Cutaneous manifestations of tuberculosis are uncommon, can be nonspecific, and may demonstrate a wide variety of morphologies, including ulcerative papules, verrucous plaques, or nodules. We report the case of a 36-year-old Moroccan man who presented with nearly 2 years of generalized folliculocentric pustules and nodules, night sweats, and weight loss. Workup revealed necrotic right axillary lymphadenopathy, multiple ill-defined hepatic lesions, and a positive interferon $-\gamma$ release assay. These findings were most consistent with papulonecrotic tuberculosis.

\section{Case Report}

A 36-year-old otherwise healthy Moroccan man presented with 2 years of fulminant, folliculocentric pustules and nodules which began on the trunk and spread to the extremities, genitals, face, and scalp, sparing the palms and soles. He reported sweats and subjective fevers, concurrent with rash onset and intermittently recurring. Over the past year, he experienced fatigue and a 10-lb weight loss. He completed a 6-month course of doxycycline which 

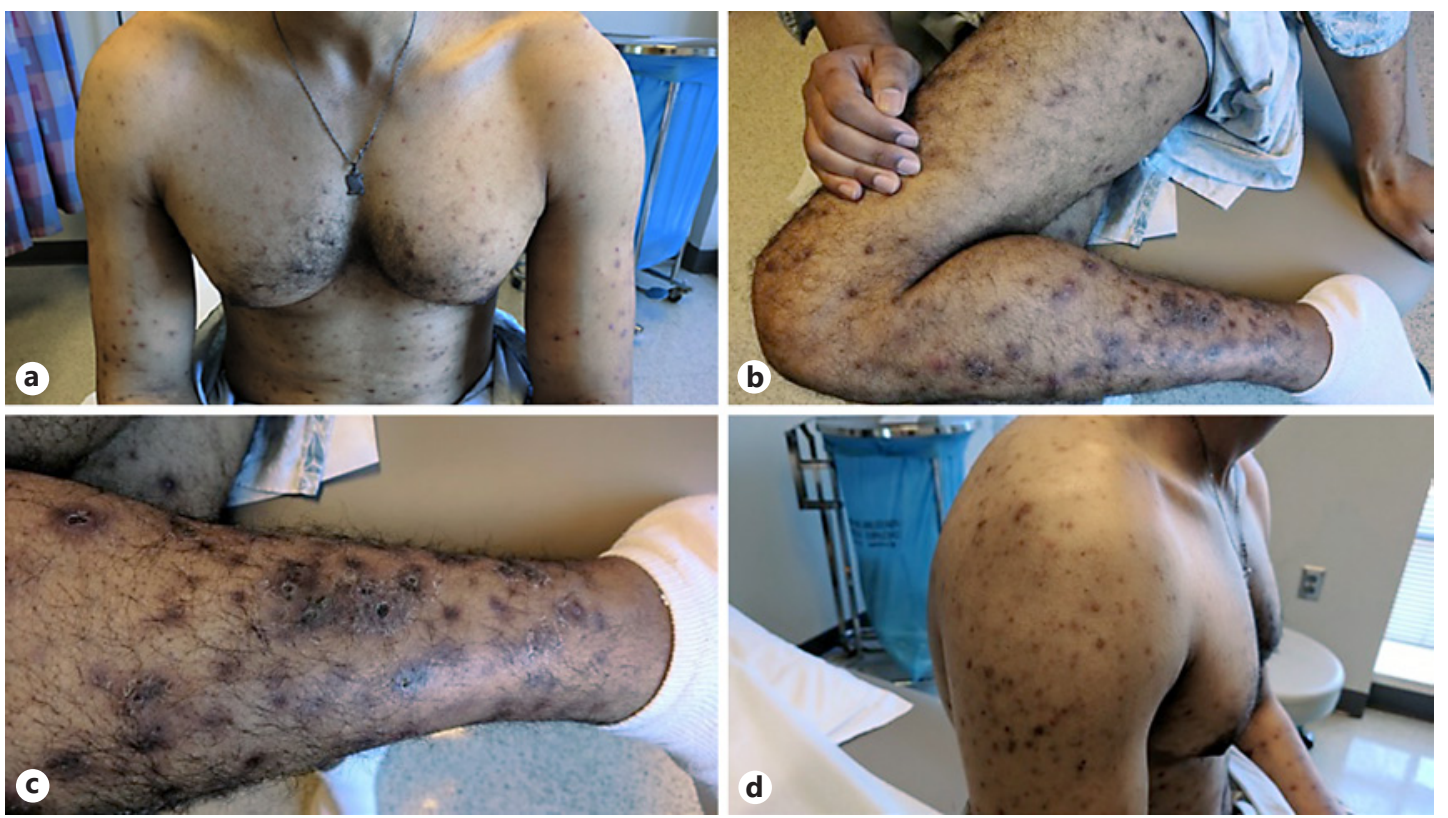

Fig. 1. Generalized folliculocentric papules and pustules were visible on the face, scalp, chest (a), bilateral lower extremities (b, c), and bilateral upper extremities (d). Examination was also notable for significant postinflammatory hyperpigmentation, which is follicularly based.

helped with rash severity, yet lesions still recurred. He applied clobetasol cream intermittently and chlorhexidine gluconate solution (Hibiclens) without sustained improvement. His labs were negative for HIV, HCV, treponemal antibody, ANA, RF, and ANCA, while his CRP was $82 \mathrm{mg} / \mathrm{L}$ (reference range $<3 \mathrm{mg} / \mathrm{L}$ ) and ESR $69 \mathrm{~mm} / \mathrm{h}$ (reference range $<15 \mathrm{~mm} / \mathrm{h}$ ). An initial punch biopsy from his left arm showed acute folliculitis; Brown Hopps stain revealed Gram-positive cocci in the follicular infundibulum.

Three months later in follow-up, his rash had worsened, with many new, generalized and painful lesions (Fig. 1). An intensive decolonization regimen was recommended, along with two systemic antibiotics (clindamycin and rifampin) pending bacterial culture results of a pustule. Bacterial culture and biopsy were repeated a month later at another hospital, yielding results again consistent with folliculitis. Oral clindamycin and rifampin were initiated. A week later, he presented to the emergency department with 2 days of severe right upper quadrant pain, with examination revealing right axillary lymphadenopathy. Abdominal CT showed multiple hepatic ill-defined hepatic lesions. Dermatology was consulted and performed two 3-mm punch biopsies of lesions identified by the patient and his wife as the newest-appearing lesions [1].

\section{Diagnosis and Clinical Course}

Histopathologic examination revealed superficial and deep necrotizing granulomatous inflammation, with a striking area of necrosis surrounding a naked hair shaft (Fig. 2). No microorganisms were detected by Brown Hopps, GMS, PAS/D, Fite, or AFB stains. Notably, his serum interferon- $\gamma$ release assay was positive. Both FNA and core biopsy of the right axillary lymph node showed a benign-appearing lymph node with necroinflammatory debris and lymphoplasmacytic infiltration. Special stains for acid-fast bacilli, fungi, bacterial, spiro- 
Morenz et al.: A 36-Year-Old Moroccan Man Presenting with Widespread Papules and Pustules
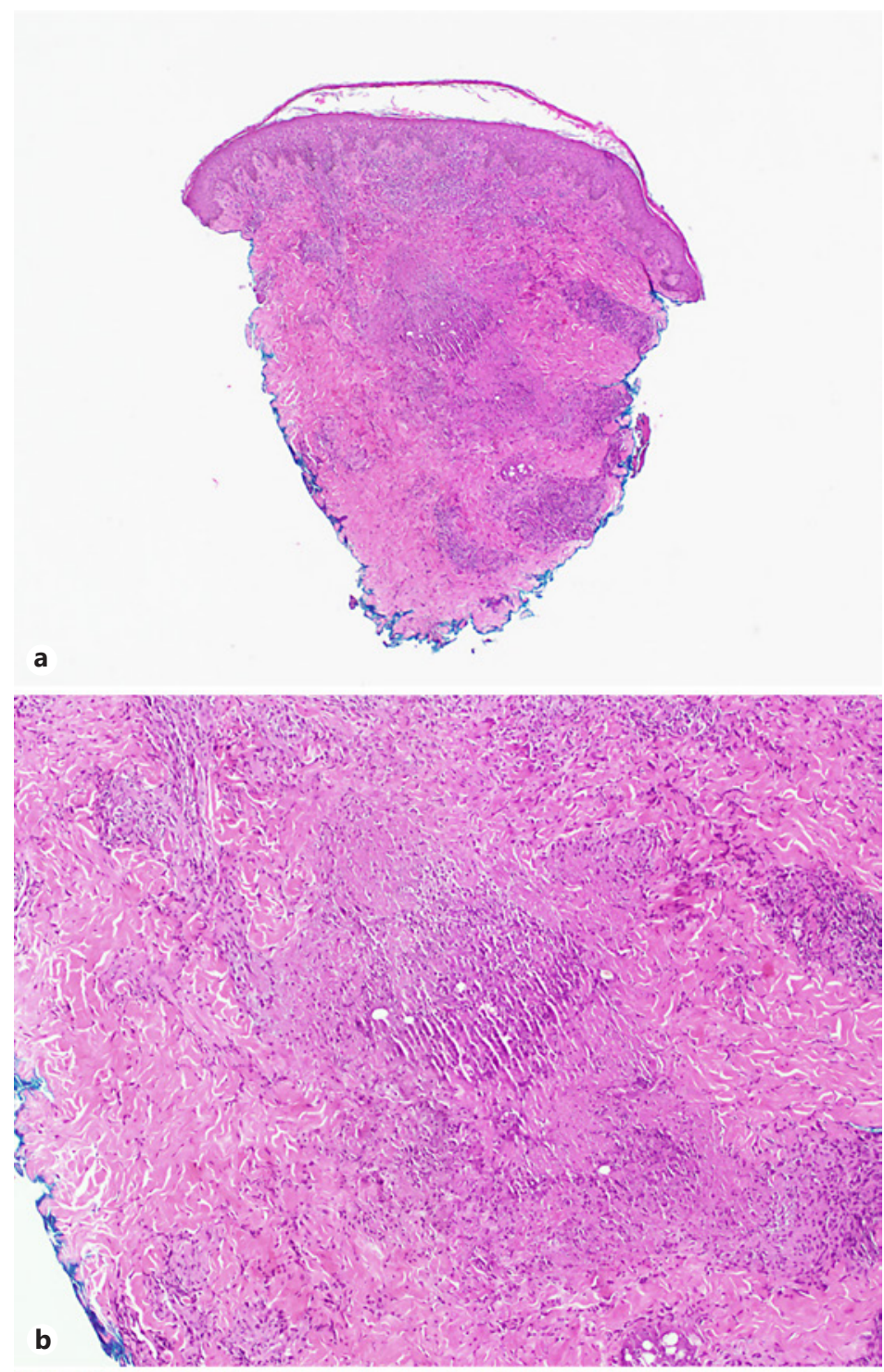

Fig. 2. Punch biopsy of the right forearm. a Low-power view of H\&E showing a superficial and deep process. $\times 40$. b, c Mediumand high-power views of H\&E, notable for superficial and deep necrotizing granulomatous inflammation. $\mathbf{b} \times 100$. $\mathbf{c} \times 400$.

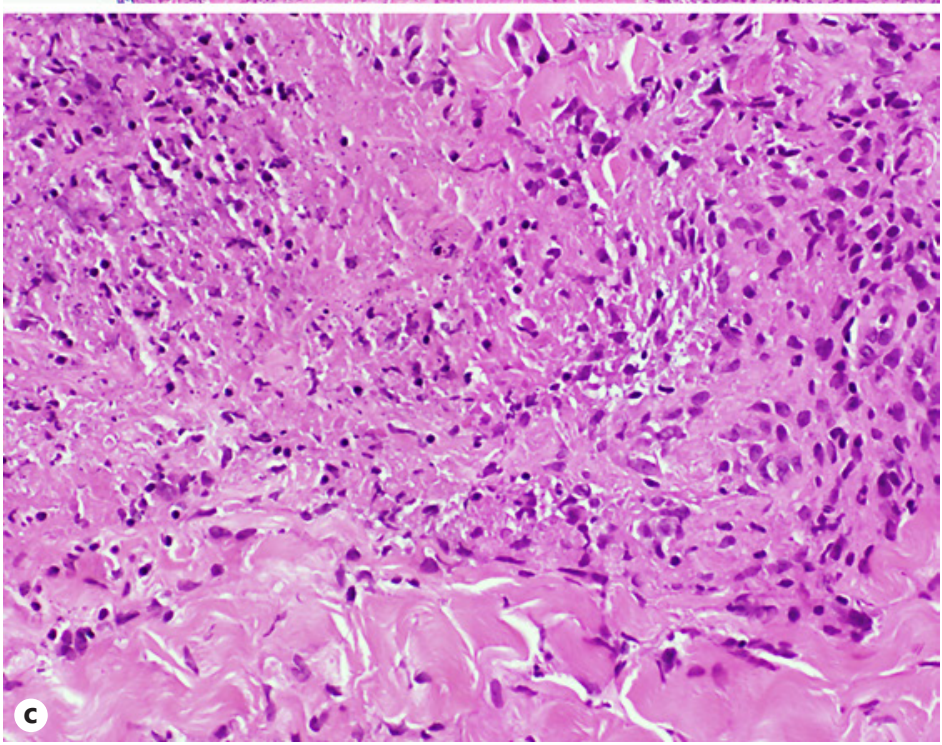


Morenz et al.: A 36-Year-Old Moroccan Man Presenting with Widespread Papules and Pustules

chetes, and antitreponemal immunohistochemistry were negative. The constellation of lymphadenopathy, hepatic lesions, and generalized ulcerative papules and nodules along with these pathology results were most consistent with papulonecrotic tuberculid tuberculosis, a variant of cutaneous tuberculosis (CTB). After pulmonary assessment showed no involvement, he was discharged home with the regimen of rifampin, isoniazid, pyrazinamide, and ethambutol.

\section{Discussion}

Extrapulmonary tuberculosis comprises approximately $10 \%$ of all tuberculosis cases, with only $1-1.5 \%$ of these patients presenting with cutaneous manifestations [2]. In developed countries, typical risk factors for CTB are immunosuppression from malnutrition, malignancy, chronic steroid use, or immunosuppressive therapy, while in developing countries, CTB occurs more often in healthy patients [3]. In Morocco, one study estimated that CTB represents $2 \%$ of all dermatologic conditions seen at a university hospital in Casablanca [4]. Cutaneous tuberculosis may demonstrate a wide variety of morphologies and is classified according to either exogenous or endogenous infection [3].

Exogenous infection occurs following direct inoculation into the skin resulting in a painless, ulcerated tuberculous chancre or tuberculous verrucosa cutis, traditionally called "anatomist's warts." Potential means of infection include tattooing or handling of infected pathologic materials. Endogenous infection, as in this patient's case, occurs due to contiguous, hematogenous, or lymphatic spread of tuberculosis. Scrofuloderma, as an example of contiguous extension, starts when an affected lymph node becomes indurated, breaks down, and spreads to the overlying skin with subsequent nodule formation. Lupus vulgaris, which has varying morphologies, most commonly presents on the head and neck and in Western countries, usually originates from an endogenous tuberculosis source. Acute disseminated miliary tuberculosis, typically secondary to hematogenous spread from active pulmonary infection, is marked by discreet pinpoint papules topped with tiny vesicles [3].

Papulonecrotic tuberculid appears as symmetrical, erythematous or violaceous papulonodular lesions which spontaneously involute, leaving depressed scars [5]. The advent of PCR detection of Mycobacterium tuberculosis DNA in these skin lesions has enabled clinicians to show that most cases of "tuberculid" lesions are actually true cutaneous tuberculosis. Due to the robust inflammatory reaction in this disseminated form of tuberculosis, acid-fast bacilli are difficult to isolate, explaining absent acid-fast bacilli in this patient's skin biopsies.

This patient's case was initially misdiagnosed as severe folliculitis, given the folliculocentric, erythematous papules and pustules. Inconsistent with this diagnosis of folliculitis was his report of several papules on the follicle-free skin of the glans penis. The systemic symptoms of night sweats and weight loss would also be unusual with folliculitis. Papulonecrotic tuberculid may also be confused with pityriasis lichenoides et varioliformis acuta (PLEVA), an uncommon T-cell lymphoproliferative disorder characterized by acute onset of erythematous papules that spontaneously resolve and recur. PLEVA may also be accompanied by fever, generalized lymphadenopathy, and malaise. PLEVA tends to present in late childhood and early adulthood, earlier than the age of our patient [6].

In terms of management, cutaneous tuberculosis is typically treated with the standard antituberculosis regimen of rifampin, isoniazid, pyrazinamide, and ethambutol. Surgical excision and reconstruction are often needed in advanced cases. Currently, no topical therapy has been shown to effectively treat any type of cutaneous tuberculosis [2]. 
Morenz et al.: A 36-Year-Old Moroccan Man Presenting with Widespread Papules and Pustules

\section{Statement of Ethics}

We thank the patient for granting written permission to publish this case and the corresponding images.

\section{Disclosure Statement}

The authors have no conflicts of interest to declare.

\section{Funding Sources}

The authors have no financial relationships relevant to this article to disclose.

\section{References}

1 Chen ST, Cahalane AM, Ryan ET, Foreman RK. Case 2-2019: A 36-year-old man with rash, abdominal pain, and lymphadenopathy. N Engl J Med. 2019 Jan;380(3):275-283.

2 van Zyl L, du Plessis J, Viljoen J. Cutaneous tuberculosis overview and current treatment regimens. Tuberculosis (Edinb). 2015 Dec;95(6):629-38.

3 Barbagallo J, Tager P, Ingleton R, Hirsch RJ, Weinberg JM. Cutaneous tuberculosis: diagnosis and treatment. Am J Clin Dermatol. 2002;3(5):319-28.

4 Zouhair K, Akhdari N, Nejjam F, Ouazzani T, Lakhdar H. Cutaneous tuberculosis in Morocco. Int J Infect Dis. 2007 May;11(3):209-12.

5 Dias MF, Bernardes Filho F, Quaresma MV, Nascimento LV, Nery JA, Azulay DR. Update on cutaneous tuberculosis. An Bras Dermatol. 2014 Nov-Dec;89(6):925-38.

6 Khachemoune A, Blyumin ML. Pityriasis lichenoides: pathophysiology, classification, and treatment. Am J Clin Dermatol. 2007;8(1):29-36. 